\title{
Avalanche Breakdown Timing Statistics for Silicon Single Photon Avalanche Diodes
}

\author{
Jonathan D. Petticrew ${ }^{(1)}$, Student Member, IEEE, Simon J. Dimler ${ }^{(1)}$, Xinxin Zhou, \\ Alan P. Morrison ${ }^{(\mathbb{1}}$, Senior Member, IEEE, Chee Hing Tan ${ }^{(\mathbb{0}}$, Member, IEEE, and Jo Shien $\mathrm{Ng}^{\mathbb{(}}{ }^{\text {, }}$ Member, IEEE
}

\begin{abstract}
Silicon-based single photon avalanche diodes (SPADs) are widely used as single photon detectors of visible and near infrared photons. There has, however, been a lack of models accurately interpreting the physics of impact ionization (the mechanism behind avalanche breakdown) for these devices. In this paper, we present a statistical simulation model for silicon SPADs that is capable of predicting breakdown probability, mean time to breakdown, and timing jitter. Our model inherently incorporates carriers' dead space due to phonon scattering and allows for nonuniform electric fields. Model validation included avalanche gain, excess noise factor, breakdown voltage, breakdown probability, and timing statistics. Simulating an n-on-p and a p-on-n SPAD design using our model, we found that the n-on-p design offers significantly improved mean time to breakdown and timing jitter characteristics. For a breakdown probability of 0.5 , mean time to breakdown and timing jitter from the n-on-p design were 3 and 4 times smaller compared to those from the p-on-n design. The data reported in this paper are available from the ORDA digital repository (DOI: 10.15131/shef.data.4823248).
\end{abstract}

Index Terms-Avalanche breakdown, avalanche photodiodes, impact ionization, jitter, silicon.

\section{INTRODUCTION}

A GROWING number of optical-based applications are sending and/or detecting optical signals at the single photon level. In applications such as Raman spectroscopy [1], optical tomography [2] and deep space optical communications [3], being able to detect weaker optical signals leads to more sensitive systems. For other applications, however, using optical signals at the single photon level is essential to the principle of operation, e.g., quantum key distribution [4] and photon-based quantum information processing [5].

Manuscript received May 31, 2017; revised October 26, 2017; accepted November 29, 2017. Date of publication December 4, 2017; date of current version December 28, 2017. This work was supported in part by the Science and Technology Facilities Council (ST/N000145/1) and in part by the Engineering \& Physical Sciences Research Council (EP/K503149/1 and EP/L505055/1). (Corresponding author: Jonathan D. Petticrew.)

J. D. Petticrew, S. J. Dimler, C. H. Tan, and J. S. Ng are with the Department of Electronic and Electrical Engineering, University of Sheffield, Sheffield S3 7HQ, U.K. (e-mail: JDPetticrew1@ sheffield.ac.uk; s.dimler@sheffield.ac. uk; c.h.tan@sheffield.ac.uk; j.s.ng@sheffield.ac.uk).

$\mathrm{X}$. Zhou was with the Department of Electronic and Electrical Engineering, University of Sheffield, Sheffield S3 7HQ, U.K. She is now with Oclaro, Northamptonshire NN12 8EQ, U.K. (e-mail: xinxin.zhou@oclaro.com).

A. P. Morrison is with the Department of Electrical and Electronic Engineering, University College Cork, Cork T12 R229, Ireland (e-mail: a.morrison@ ucc.ie).

Digital Object Identifier 10.1109/JSTQE.2017.2779834
Single-photon-level signals can be detected using various detectors including Si-based Single Photon Avalanche Diodes (SPADs) [6], InGaAs-based SPADs [7], Superconducting Nanowire Single Photon Detectors (SNSPDs) [8], and electron-Avalanche Photodiodes (e-APDs) [9]. Despite being limited to optical signals with wavelengths $<1 \mu \mathrm{m}$, Si-based SPADs are widely employed. Si-based SPADs have a relatively high operating temperature, compared to SNSPDs that require very low operating temperatures of a few Kelvin. They also have low false counts, with $800 \mathrm{cps}$ for Si SPADs (50 $\mu \mathrm{m}$ diameter) [10] compared to 500 Mcps for InGaAs-based SPADs (size not specified) [11], at 55\% detection efficiency.

The importance of Si-based SPADs gave rise to development of simulation models for Si SPADs. The key performance parameters investigated are (i) breakdown probability, $P_{b}$, which determines the detection efficiency of a SPAD, and (ii) timing jitter, usually defined as Full-Width at Half-Maximum (FWHM) of time to detect avalanche breakdown. Since both parameters are entirely determined by the impact ionization process in $\mathrm{Si}$, a valid model must contain accurate information of the impact ionization process at electric field magnitudes relevant to $\mathrm{Si}$ SPADs to yield accurate simulations of $P_{b}$ and timing jitter.

To accurately describe the impact ionization process, the carriers' history, including dead space, which is significant in submicron avalanche regions (common in Si-based SPADs with small timing jitter), must be included [12]. Furthermore, for devices with highly non-uniform electric fields (again common in Si-based SPADs), careful incorporation of history-dependent treatment into the impact ionization process is essential.

The early Si-based SPAD simulation work [13], [14] did not include the carriers' dead space, because they were completed before the significance of dead space had been fully realized and investigated by Avalanche Photodiode (APD) researchers. Since then, coupled integral equations for simulations of breakdown probability accounting for dead space have been reported [15], [16]. Later, mean time to breakdown, $T_{b}$, and timing jitter were simulated in a study that included dead space but used only constant electric fields [17]. This was followed by a Si SPAD model by Ingargiola et al. [18], which included the carriers' dead space and tried to accommodate non-uniform electric fields. However, their model was only able predict the breakdown voltages, $V_{b}$, of two Si SPADs, after adjusting ionization coefficients [18]. In summary, none of the models mentioned above offer sufficient validation required for accurate Si SPAD simulations of probability and timing statistics of avalanche breakdowns. 
Since then, a Simple Monte Carlo (SMC) model [19] for simulating avalanche gain, $M$, and excess noise factor, $F$, as function of reverse bias, $V$, was developed for Si APDs [20]. A SMC model contains far less band structure details, and so requires far shorter computation-time, compared to standard Full Band Monte Carlo models. Crucially, SMC models include carrier scattering mechanisms (including impact ionization) updated on a femtosecond timescale, so it can handle highly non-uniform electric fields.

Reported SMC models underwent extensive benchmarking with experimental data on impact ionization, covering a wide range of electric fields. For example, the Si SMC model in [20] was benchmarked against experimental $V_{b}, M(V)$, and $F(M)$ of five Si diodes (with breakdown electric fields from 230 to $830 \mathrm{kV} \cdot \mathrm{cm}^{-1}$ ), including a p-n diode with a highly non-uniform electric field profile.

Building on [20], in this work we report an SMC model for Si SPAD simulations. In addition to simulating $V_{b}, M(V)$, and $F(M)$, as for the typical SMC models, our SMC model for Si SPADs provides breakdown probability, mean time to breakdown, and timing jitter as functions of reverse bias. In this work, the time to breakdown is defined as the time elapsed between carrier injection and current threshold being reached.

\section{MODEL}

As in the first SMC models reported by Plimmer [19], our Si SMC model incorporates two main carrier scattering mechanisms; effective intervalley phonon scattering (phonon absorption and phonon emission) and impact ionization. In the simulated SPAD, a carrier drifts in the depletion region of the device and gains energy from the electric field, $\mathfrak{I}$. At the end of a free flight (typically femtoseconds long), the carrier can undergo either a phonon scattering or an impact ionization event, as determined by a random selection. Our model uses the same phonon scattering rates and ionization rates as the Si SMC model in [20], which was validated not only with experimental impact ionization-related data but also electron and hole drift velocities versus uniform electric field.

SMC models record the carriers' positions to produce distributions of avalanche gain (giving mean gain and excess noise factors). The instantaneous currents contributed by each of the carriers can also be computed in an SMC model [21], by applying Ramo's theorem [22], $I(t)=q v / w$, where $q$ is the charge of an electron, $v$ is the instantaneous velocity of the carrier and $w$ is the depletion width. The Si SMC model of this work computes the carrier's position and velocity as well as the resultant instantaneous current. Each trial outputs the total current, from which the associated avalanche gain, $m$, is computed. From a histogram of $m, M=\langle m\rangle$ and $F=\left\langle m^{2}\right\rangle /\left\langle m^{2}\right\rangle$ can be obtained. The total current also gives the time taken to reach breakdown, if the current threshold (set to $0.1 \mathrm{~mA}$ ) is reached. The level of $0.1 \mathrm{~mA}$ (generating a $5 \mathrm{mV}$ voltage drop across a $50 \Omega$ resistance) is detectable experimentally using an avalanche quenching/sensing circuit [23].

For a given set of simulation conditions, the number of trials reaching breakdown and the associated time to breakdown were

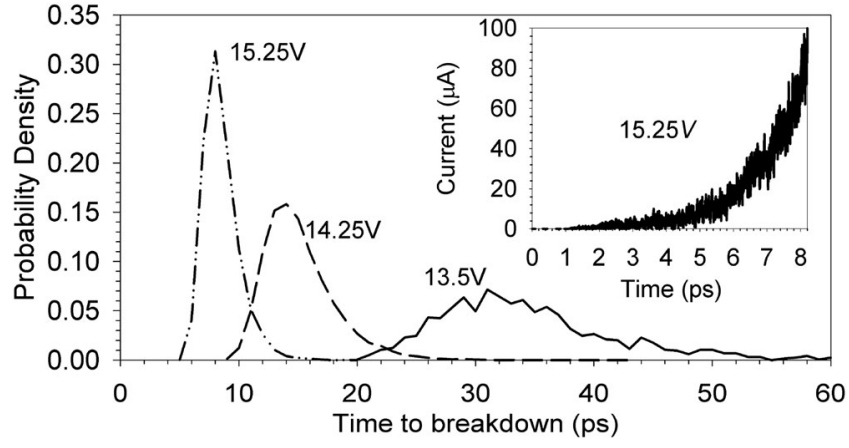

Fig. 1. Probability density functions of time to breakdown for 10000 events for a p-i-n device with a depletion width of $0.26 \mu \mathrm{m}$ at $V=13.5,14.25$ and $15.25 \mathrm{~V}\left(P_{b}=0.56,0.87\right.$ and 0.98$)$. Inset is the current versus time data from a single trial of the device at $15.25 \mathrm{~V}$.

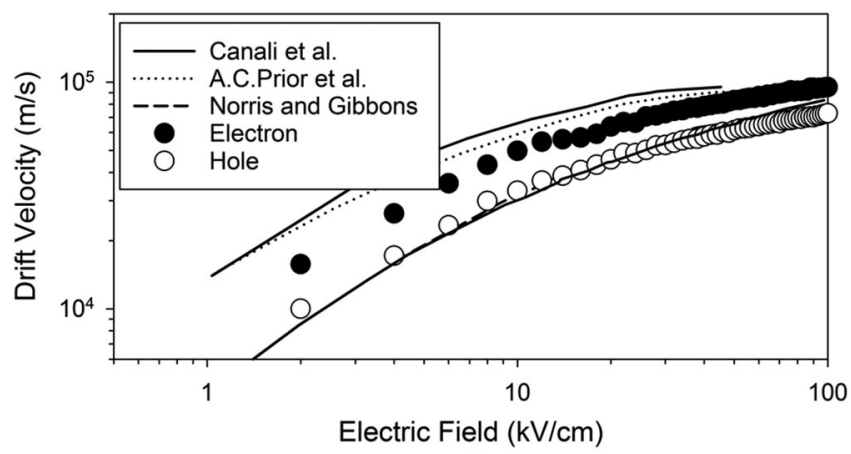

(a)

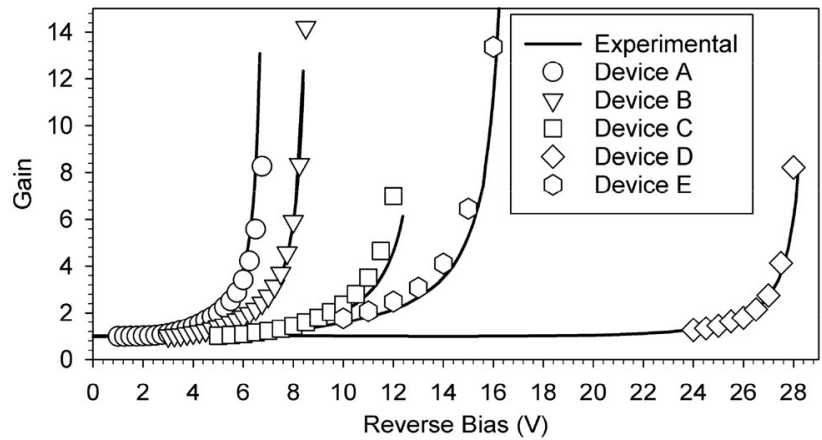

(b)

Fig. 2. (a) Drift velocity versus electric field from the SMC model and refs [24]-[26], and (b) $M(V)$ obtained from instantaneous currents of the SMC model (symbols) and experimental data (lines) for device A to $\mathrm{E}$ from [27].

recorded. The ratio of the former to the total number of trials gives $P_{b}$. The latter yields a histogram of time to breakdown, $t_{b}$, which in turn produces mean time to breakdown, $T_{b}$, and timing jitter due to the stochastic nature of impact ionization.

The probability density functions (PDFs) of $t_{b}$ of a p-i-n device with a depletion width of $0.26 \mu \mathrm{m}$ at $V=13.5,14.25$ and $15.25 \mathrm{~V}\left(P_{b}=0.56,0.87\right.$ and 0.98$)$ are shown in Fig. 1 , as examples of SPAD timing information available from the model. Each PDF was generated using the trials that reached avalanche breakdown out of a total of 10000 trials. As $V$ increases, which increases $P_{b}$, the avalanche process speeds up and there is less 
TABLE I

Device A to E [27] Used In Avalanche Gain AND EXCESS NoISE FACTORS VALIDATION

\begin{tabular}{lccccc}
\hline \hline Device & Structure $W(\mu \mathrm{m})$ & $\begin{array}{c}\mathrm{N}_{\mathrm{p}}(\times \\
\left.10^{18} \mathrm{~cm}^{-3}\right)\end{array}$ & $\begin{array}{c}\mathrm{N}_{\mathrm{i}}(\times \\
\left.10^{16} \mathrm{~cm}^{-3}\right)\end{array}$ & $\begin{array}{c}\mathrm{N}_{\mathrm{n}}(\times \\
\left.10^{18} \mathrm{~cm}^{-3}\right)\end{array}$ \\
\hline A & P-I-N & 0.082 & 2.5 & 2.0 & 4.0 \\
B & P-I-N & 0.13 & 3.0 & 2.0 & 3.0 \\
C & P-I-N & 0.26 & 3.0 & 2.0 & 3.0 \\
D & N-I-P & 0.82 & 6.0 & 1.8 & 0.5 \\
E & P-N & - & 7.5 & - & 0.075 \\
\hline \hline
\end{tabular}

variation in $t_{b}$. An example of instantaneous current from a single trial that reached $0.1 \mathrm{~mA}$ is shown in the inset of Fig. 1. The carrier transit time for the $0.26 \mu \mathrm{m}$ device is $\sim 0.26 \mathrm{ps}$, assuming a carrier saturation velocity of $10^{5} \mathrm{~m} \cdot \mathrm{s}^{-1}$. Hence, the peaks at 8,14 , and 31 ps in Fig. 1 correspond to approximately 3,5 , and 11 times the carrier's transit time, respectively.

\section{VALIDATION}

Since the model of this work builds on [20], it was successfully validated with the usual comparisons of simulated results and experimental results for (i) electron and hole drift velocities versus electric field [24]-[26] shown in Fig. 2, as well as (ii) $V_{b}$, $M(V)$, and $F(M)$ [27], as expected. The number of trials used for a given set of conditions was 10000 . For part (ii), three p-i-n devices, one n-i-p device and a p-n device reported in [27] were used and their structure details are summarized in Table I. The unintentionally doped layers were n-type. The simulations used pure electron injection conditions for the $\mathrm{p}-\mathrm{i}-\mathrm{n}$ and $\mathrm{p}-\mathrm{n}$ devices, and pure hole injection conditions for the $n-i-p$ device. These results suggest the SMC is capable of predicting behavior in devices with a highly non-uniform electric field.

$M(V)$ from the simulated instantaneous current are compared with the experimental data [27] for the five devices in Fig. 2, and $F(V)$ (not shown here). Fitting $1 / M$ against $V$ (not shown here) yielded values of $V_{b}$ of 7.37, 8.99, 13.1,29.3 and $17 \mathrm{~V}$ for devices A-E, respectively. The agreement in Fig. 2 demonstrates the Si SMC model's ability to accurately simulate instantaneous currents for linear mode APDs. With model validations for APDs completed, the Si SMC model was then used to simulate SPAD devices at $V>V_{b}$.

Simulated $P_{b}$ versus reverse bias characteristics for devices A-E are shown in Fig. 3, with the $V_{b}$ from Fig. 2(b) included as reference values. For all devices, $P_{b}(V)$ rises from zero at $V=V_{b}$, approaching unity as $V$ increases, as expected from the established theory [15].

The SMC $P_{b}(V)$ results are also compared with those calculated using recurrence equations by McIntyre [15, Eqn. 29], as shown in Fig. 4. The device structure used was an ideal p-i-n diode with $1.0 \mu \mathrm{m}$ i-region width, $w$. The recurrence equations require PDFs of electron and hole impact ionization path lengths, $h_{e}(\xi)$ and $h_{h}(\xi)$, as inputs. Hard dead space approximations [28] were used so

$$
h_{e}(\xi)= \begin{cases}0 & \xi<d_{e} \\ \alpha^{*} \exp \left(-\alpha^{*}\left(\xi-d_{e}\right)\right) & \xi \geq d_{e}\end{cases}
$$

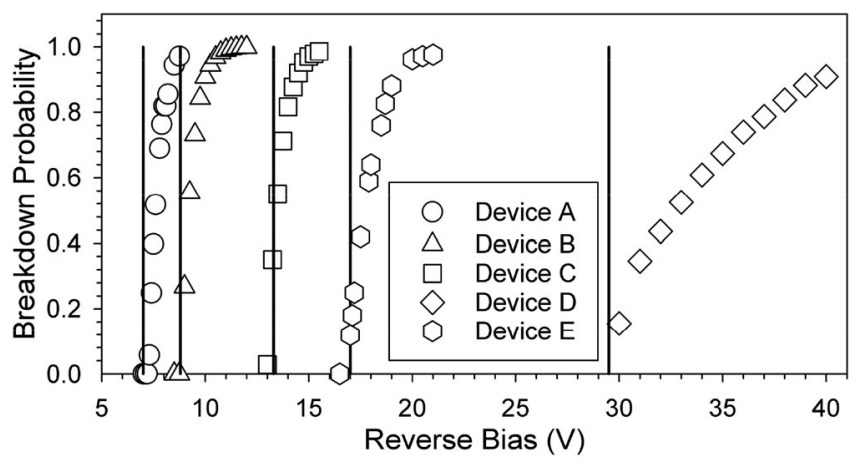

Fig. 3. Simulated breakdown probability versus reverse bias using an avalanche current threshold of $0.1 \mathrm{~mA}$. The $V_{b}$ values for device A to E from Fig. 2(b) are included as vertical lines for reference.

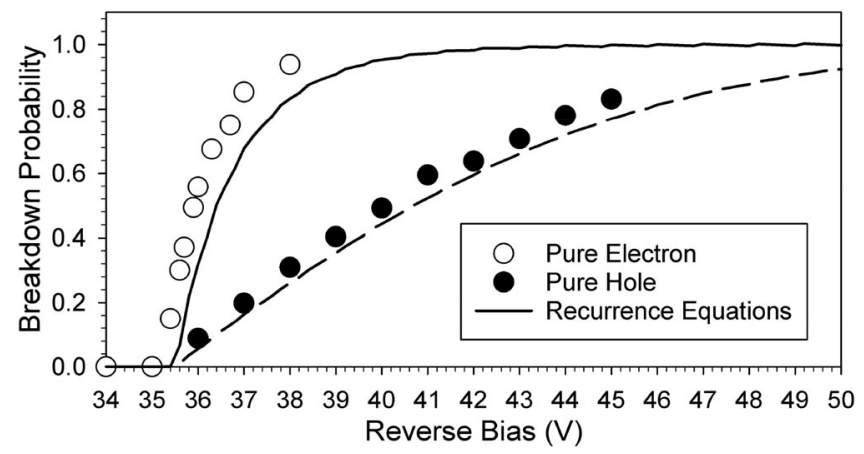

Fig. 4. Comparison of breakdown probability versus reverse bias characteris tics for a $1.0 \mu \mathrm{m}$ ideal p-i-n diode from the SMC model (symbols) and recurrence equations by McIntyre [15] (lines).

where $\alpha^{*}$ and $d_{e}$ are the electron's effective ionization coefficient and dead space, respectively. A similar expression was used for $h_{h}(\xi)$, by replacing $\alpha^{*}$ and $d_{e}$ with the hole's effective ionization coefficient and dead space, $\beta^{*}$ and $d_{h}$, respectively. Dead spaces were given by $d_{e(h)}=E_{\text {the(h) }} / q \mathfrak{\Im}$, where $E_{t h e(h)}$ is ionization threshold energy for electron (hole) in $\mathrm{eV}$ and is the electric field in $\mathrm{V} \cdot \mathrm{m}^{-1}$. Due to a lack of readily available values for $\alpha^{*}(\Im), \beta^{*}(\Im), E_{t h e}$ and $E_{t h h}$, these were obtained from separate simulations of ionization path lengths using the Si SMC model. Values of $E_{\text {the }}$ and $E_{t h h}$ were found to be 2.18 and $3.41 \mathrm{eV}$, respectively. For electric fields from 300 to $800 \mathrm{kV} \cdot \mathrm{cm}^{-1}$, we found that

$$
\alpha^{*}(\Im)=5.0 \times 10^{7} \exp \left(-\left(8.0 \times 10^{7} / \Im\right)^{1.28}\right),
$$

and

$$
\beta^{*}(\Im)=4.4 \times 10^{7} \exp \left(-\left(9.4 \times 10^{7} / \Im\right)^{1.65}\right)
$$

The comparison in Fig. 4 shows reasonable agreement, with a maximum variation of $21 \%$, between results from the SMC model and the recurrence equations, for an electron-hole-pair injected at the junction of $\mathrm{p}^{+} / \mathrm{i}$ (pure electron injection) or $\mathrm{i} / \mathrm{n}^{+}$ (pure hole injection). For a given reverse bias, $P_{b}$ is higher when pure electron injection is used, compared to pure hole 


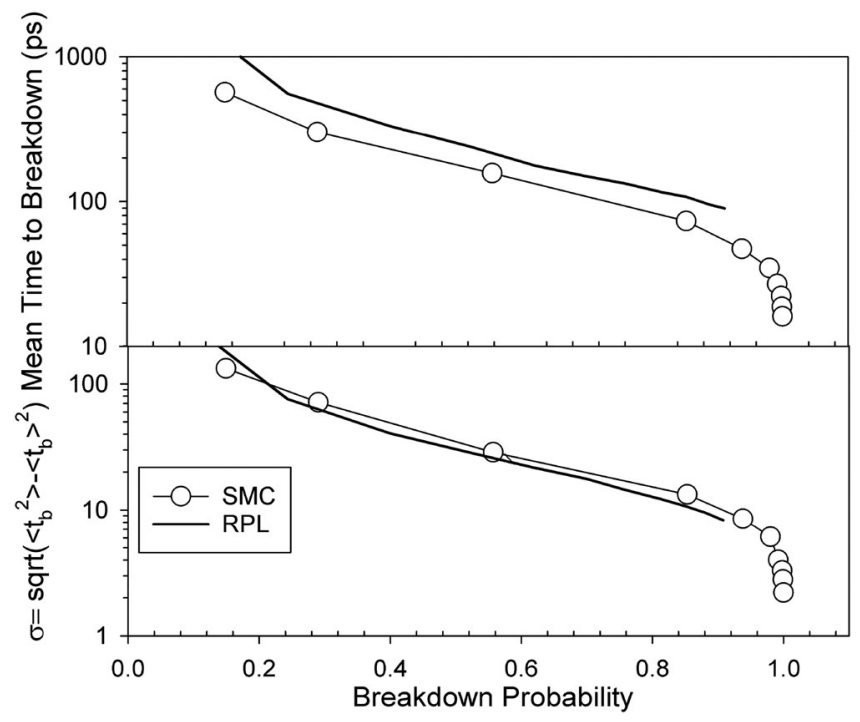

Fig. 5. Simulated mean (top) and standard deviation (bottom) of $t_{b}$ for a $1.0 \mu \mathrm{m}$ ideal p-i-n diode, by the SMC model and a Random Path Length model, which assumes constant carrier velocities [17].

injection. This is expected from existing knowledge of avalanche breakdown probability [13] because $\alpha^{*}>\beta^{*}$ in $\mathrm{Si}$.

Timing information of the same $w=1.0 \mu \mathrm{m}$ ideal $\mathrm{p}-\mathrm{i}-\mathrm{n}$ diode from the SMC model is presented in Fig. 5, in the form of $T_{b}$ and standard deviation of $t_{b}$. Using standard deviations, instead of FWHM, for this particular figure allows direct comparison with timing data from simpler models assuming constant carrier velocities, such as the Random Path Length (RPL) model [17]. Data from ref [17], which is included in Fig. 5, assumed a constant drift velocity of $10^{5} \mathrm{~m} \cdot \mathrm{s}^{-1}$ for both electrons and holes. Timing jitter predicted by both models is similar, but the RPL model overestimates $T_{b}$. The discrepancy in $T_{b}$ could be caused by the RPL model's assumption of a constant carrier velocity for all carriers, which underestimates the velocity of the ionizing carriers [21]. It is noted that highly non-uniform electric fields in actual Si SPADs are likely to introduce further errors in the timing information from simpler models.

\section{EFFEcts of SPAD DESIGN ON TIMING PERFoRMAnCE}

The SMC model was then used to investigate effects of SPAD design on avalanche breakdown timing performance. Using an $\mathrm{n}^{+}-\mathrm{p}-\mathrm{p}^{-}-\mathrm{p}^{+}$structure, we consider two Si SPAD designs, which have either the $\mathrm{n}^{+}$-region or the $\mathrm{p}^{+}$-region on the top of the semiconductor's surface. The former (n-on-p design) is the orientation used in commercial SPADs, whereas the latter ( $p$ on-n design) will require changes in device fabrication method (though within the technical ability of current semiconductor technologies). Both designs have identical breakdown voltage of $27 \mathrm{~V}$ and their electric field profiles at this voltage are shown in Fig. 6. It was assumed that incident photons enter from the top of the semiconductor's surface in both designs and are fully absorbed in the top $\mathrm{n}^{+}$or $\mathrm{p}^{+}$region. Hence this investigation also covers comparison of pure hole injection ( $n$-on-p design) and pure electron injection (p-on-n design).

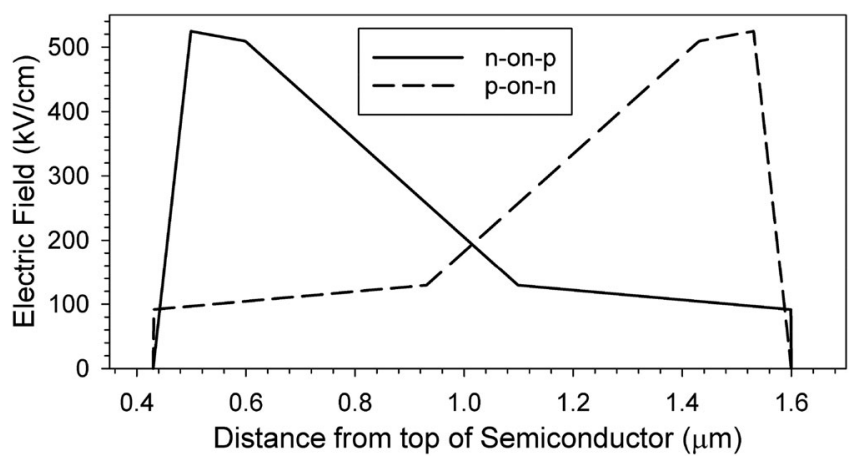

Fig. 6. Electric field profiles at $V_{b}$ for n-on-p and p-on-n SPAD designs.

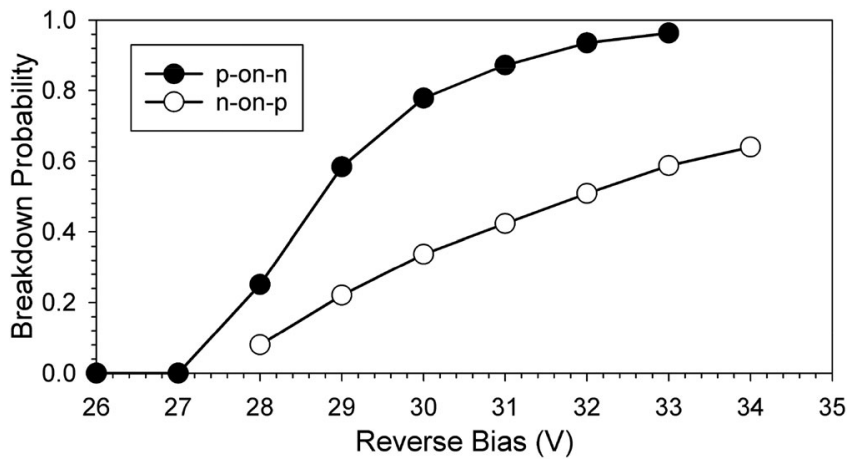

Fig. 7. Breakdown probability versus reverse bias for n-on-p and p-on-n SPAD designs.

Simulations of $P_{b}, T_{b}$, and timing jitter were carried out for both designs. Results of $P_{b}$ versus reverse bias and timing statistics versus $P_{b}$ are plotted in Figs. 7 and 8 , respectively. In Fig. 7, for a given reverse bias, avalanche breakdown is less likely to occur in the n-on-p design than the p-on-n design. This is because the former is reliant on holes, which are less likely to undergo impact ionization, to trigger avalanche breakdown. The lower $P_{b}$ in the n-on-p design is reflected in the use of reverse bias $>1.2 V_{b}$ in SPADs to achieve good $P_{b}$ (e.g., [29]). Using reverse bias well above $V_{b}$ is practical for Si n-on-p SPADs because of their relatively low false counts. It is however less appropriate for SPADs with false counts that rise rapidly with reverse bias.

Fig. 8 shows that, at a given $P_{b}$, the n-on-p design provides significantly more desirable avalanche breakdown timing statistics (faster and less variable), compared to the p-on-n design. For example, at $P_{b}=0.5$, the n-on-p design has a mean time to breakdown and timing jitter that are 3 and 4 times smaller, respectively, than those from the p-on-n design. Note that our observation is distinct from and does not contradict ref [17], which found that, for a given $P_{b}$, both mean time to breakdown and timing jitter decrease when ionization coefficients of the avalanche material become similar.

It is unsurprising to have closely related magnitudes of $T_{b}$ and timing jitter, so a small $T_{b}$ for the n-on-p design is associated with a small timing jitter. However, the large difference between timing statistics from the two designs (or carrier injection conditions) has not been reported in design or simulation 


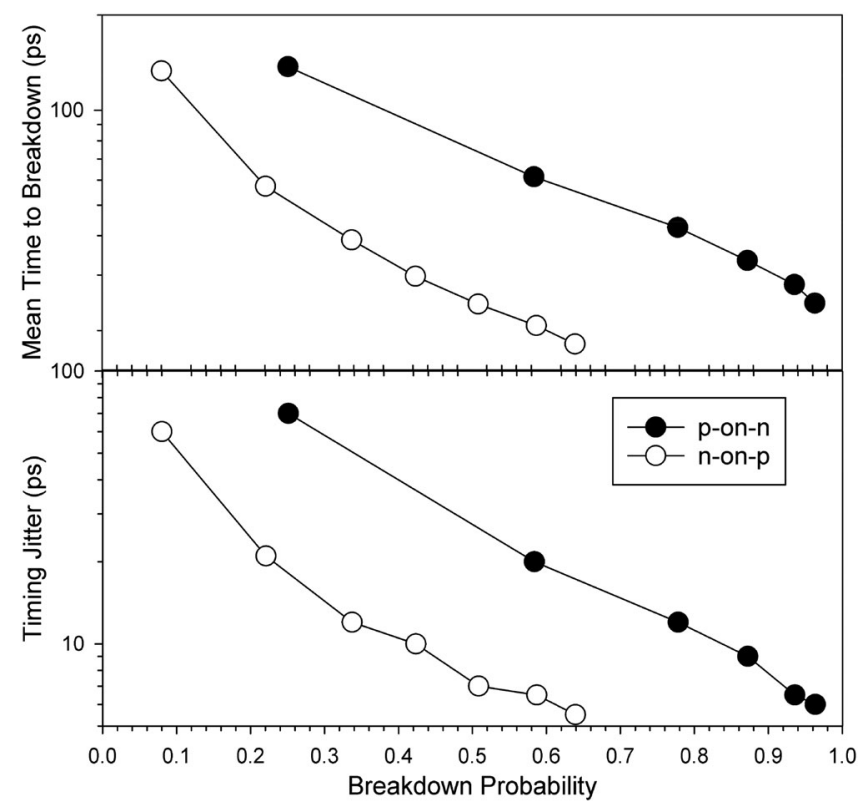

Fig. 8. Mean time to breakdown (top) and timing jitter (bottom) versus $P_{b}$ simulated for the two SPAD designs.

studies and is therefore worth further investigation. Given the gradual rise of dark count rate in a well-designed Si SPAD the adoption of n-on-p design (particularly for detection of short wavelength such as UV) could provide significant improvement in the timing statistics.

To obtain $P_{b}=0.5$, the n-on-p and p-on-n design require applied reverse bias of $32.0\left(1.19 V_{b}\right)$ and $28.7 \mathrm{~V}\left(1.06 V_{b}\right)$, with corresponding peak electric fields of 563 and $535 \mathrm{kV} \cdot \mathrm{cm}^{-1}$, respectively. For the SPAD to quickly reach breakdown $(0.1 \mathrm{~mA}$ in our simulations), the carriers need to build up energy quickly (by drifting along the electric field) and avoid losing energy or preferred trajectory (by encountering phonon scattering events). Due to lower electric field, carriers in the p-on-n design need to travel a longer distance to build up the same energy, compared to carriers in the n-on-p design.

In addition, carriers with low energy are more likely to encounter scattering events other than impact ionization. Phonon emission events delay impact ionization events, preventing rapid build up of instantaneous currents to trigger avalanche breakdown. From SMC simulations, we found mean numbers for phonon emission, phonon absorption and impact ionization for the p-on-n design that were 2.7, 3.0 and 1.6 times larger than those for the n-on-p design. Repeating the comparison at $P_{b}=0.6$ gave the same factors. In the p-on-n design, the carriers therefore had to have many more impact ionization events over a longer duration, before eventually reaching the current threshold. Hence, the differences in avalanche breakdown timing statistics of the n-on-p and p-on-n designs originate from the different electric field profiles required to produce the same breakdown probability.

While this model focuses on the contributions to timing jitter arising from the impact ionization mechanism, there exist other mechanisms that may impair the avalanche timing performance.
The current model could be expanded to include them, at the expense of simulation time. One cause of increased jitter is the absorption of photons at the edge of the SPAD's active region, resulting in avalanche pulses with timing performance very different from those triggered by photon absorption in the central active region [14]. This contribution can be significantly reduced by modern SPAD designs, which incorporate metal rings covering the periphery of the implanted active area [30]. Timing jitter contributions also arise from lateral carrier spreading through space-charge effects and photon emission from hot carriers. The former is significant at avalanche currents greater than the $0.1 \mathrm{~mA}$ current threshold used in this work, whereas the latter was found to be relatively insignificant [14].

\section{CONCLUSION}

We have developed a simulation model for predicting avalanche breakdown probability, mean time to breakdown, and timing jitter for Si single photon avalanche diodes. The only required input is the electric field profile. The validations covered both APD and SPAD related performance parameters. Using this model, we show that there are clear differences in avalanche breakdown timing performance solely due to whether a hole or an electron was injected into the SPAD. For a given breakdown probability, Si SPADs achieve much faster and less variable time to breakdown, when photons are absorbed within the n-region, i.e., holes (the carrier less likely to cause impact ionization) are injected in the SPADs.

\section{REFERENCES}

[1] D. V. Martyshkin, R. C. Ahuja, A. Kudriavtsev, and S. B. Mirov, "Effective suppression of fluorescence light in Raman measurements using ultrafast time gated charge coupled device camera," Rev. Sci. Instrum., vol. 75, no. 3, pp. 630-635, 2004.

[2] D. A. Boas et al., "Imaging the body with diffuse optical tomography," IEEE Signal Process. Mag., vol. 18, no. 6, pp. 57-75, Nov. 2001.

[3] D. M. Boroson and B. S. Robinson, "The Lunar laser communication demonstration: NASA's first step toward very high data rate support of science and exploration missions," Space Sci. Rev., vol. 185, no. 1, pp. 115-128, Dec. 2014

[4] N. Gisin, G. Ribordy, W. Tittel, and H. Zbinden, "Quantum cryptography," Rev. Mod. Phys., vol. 74, no. 1, pp. 145-195, 2002.

[5] A. Aguado et al., "Secure NFV orchestration over an SDN-Controlled optical network with time-shared quantum key distribution resources," $J$. Lightw. Technol., vol. 35, no. 8, pp. 1357-1362, Apr. 2017.

[6] A. Lacaita, M. Ghioni, and S. Cova, "Double epitaxy improves singlephoton avalanche diode performance," Electron. Lett., vol. 25, no. 13, pp. 841-843, Jun. 1989.

[7] J. Zhang, M. A. Itzler, H. Zbinden, and J.-W. Pan, "Advances in InGaAs/InP single-photon detector systems for quantum communication," Light Sci. Appl., vol. 4, no. 5, May 2015, Art. no. e286.

[8] X. Hu, Y. Cheng, C. Gu, X. Zhu, and H. Liu, "Superconducting nanowire single-photon detectors: Recent progress," Sci. Bull., vol. 60, no. 23, pp. 1980-1983, Dec. 2015.

[9] X. Sun, J. B. Abshire, and J. D. Beck, "HgCdTe e-APD detector arrays with single photon sensitivity for space lidar applications," Proc. SPIE, vol. 9114, May 2014, Art. no. 91140K.

[10] PDM-R Series Datasheet, 2nd ed., Micro Photon Devices, Bolzano, Italy, Dec. 13, 2017.

[11] L. C. Comandar et al., "Gigahertz-gated InGaAs/InP single-photon detector with detection efficiency exceeding $55 \%$ at 1550 nm," J. Appl. Phys., vol. 117, no. 8, Feb. 2015, Art. no. 083109.

[12] G. J. Rees and J. P. R. David, "Nonlocal impact ionization and avalanche multiplication,” J. Phys. D., vol. 43, no. 24, Jun. 2010, Art. no. 243001 
[13] W. G. Oldham, R. R. Samuelson, and P. Antognetti, "Triggering phenomena in avalanche diodes," IEEE Trans. Electron Devices, vol. ED-19, no. 9 , pp. 1056-1060, Sep. 1972.

[14] A. Spinelli and A. L. Lacaita, "Physics and numerical simulation of single photon avalanche diodes," IEEE Trans. Electron Devices, vol. 44, no. 11 , pp. 1931-1943, 1997.

[15] R. J. McIntyre, "A new look at impact ionization-Part I: A theory of gain, noise, breakdown probability, and frequency response," IEEE Trans. Electron Devices, vol. 46, no. 8, pp. 1623-1631, Aug. 1999.

[16] M. M. Hayat et al., "Breakdown probabilities for thin heterostructure avalanche photodiodes," IEEE J. Quantum. Electron., vol. 39, no. 1, pp. 179-185, Jan. 2003.

[17] C. H. Tan, J. S. Ng, G. J. Rees, and J. P. R. David, "Statistics of avalanche current buildup time in single-photon avalanche diodes," IEEE J. Sel. Topics Quantum Electron., vol. 13, no. 4, pp. 906-910, Jul./Aug. 2007.

[18] A. Ingargiola et al., "Avalanche buildup and propagation effects on photontiming jitter in Si-SPAD with non-uniform electric field," Proc. SPIE, vol. 7320, May 2009, Art. no. 73200K.

[19] S. A. Plimmer, J. P. R. David, D. S. Ong, and K. F. Li, "A simple model for avalanche multiplication including deadspace effects," IEEE Trans. Electron Devices, vol. 46, no. 4, pp. 769-775, Apr. 1999.

[20] X. Zhou, J. S. Ng, and C. H. Tan, "A simple Monte Carlo model for prediction of avalanche multiplication process in silicon," J. Instrum., vol. 7, no. 08, 2012, Art. no. P08006.

[21] P. J. Hambleton, B. K. Ng, S. A. Plimmer, J. P. R. David, and G. J. Rees, "The effects of nonlocal impact ionization on the speed of avalanche photodiodes," IEEE Trans. Electron Devices, vol. 50, no. 2, pp. 347-351, Feb. 2003.

[22] S. Ramo, "Currents induced by electron motion," Proc. IRE, vol. 27, no. 9, pp. 584-585, Sep. 1939.

[23] I. Rech, D. Resnati, A. Gulinatti, M. Ghioni, and S. Cova, "Self- suppression of reset induced triggering in picosecond SPAD timing circuits," Rev Sci. Instrum., vol. 78, no. 8, 2007, Art. no. 086112.

[24] C. Canali, G. Ottaviani, and A. A. Quaranta, "Drift velocity of electrons and holes and associated anisotropic effects in silicon," J. Phys. Chem. Solids, vol. 32, no. 8, pp. 1707-1720, 1971

[25] A. C. Prior, "The field-dependence of carrier mobility in silicon and germanium," J. Phys. Chem. Solids, vol. 12, no. 2, pp. 175-180, 1960.

[26] C. B. Norris and J. F. Gibbons, "Measurement of high-field carrier drift velocities in silicon by a time-of-flight technique," IEEE Trans. Electron Devices, vol. ED-14, no. 1, pp. 38-43, Jan. 1967.

[27] C. H. Tan, "Measurements of excess avalanche noise in sub-micron Si and $\mathrm{Al}_{0.6} \mathrm{Ga}_{0.4} \mathrm{As}$ avalanche photodiodes," Ph.D. dissertation, Univ. Sheffield, Sheffield, U.K., 2001.

[28] M. M. Hayat, B. E. A. Saleh, and M. C. Teich, "Effect of dead space on gain and noise of double-carrier-multiplication avalanche photodiodes," IEEE Trans. Electron Devices, vol. 39, no. 3, pp. 546-552, Mar. 1992.

[29] E. A. G. Webster, L. A. Grant, and R. K. Henderson, "A high-performance single-photon avalanche diode in 130-nm CMOS imaging technology," IEEE Electron Device Lett., vol. 33, no. 11, pp. 1589-1591, Nov. 2012.

[30] F. Acerbi et al., "High detection efficiency and time resolution integratedpassive-quenched single-photon avalanche diodes," IEEE J. Sel. Topics Quantum Electron., vol. 20, no. 6, pp. 268-275, Nov. 2014.
Jonathan D. Petticrew received the M.Phys. degree in physics in 2015 from the University of Sheffield, Sheffield, U.K., where he is currently working toward the $\mathrm{Ph} . \mathrm{D}$. degree on the design and fabrication of single photon detectors.

Simon J. Dimler received the M.Eng. and Ph.D. degrees in electronic and electrical engineering from the University of Sheffield, Sheffield, U.K. His current research interests include development of single photon detectors and instrumentation for the characterization of photodetectors.

Xinxin Zhou received the M.Sc. and Ph.D. degrees in electronic engineering from the University of Sheffield, Sheffield, U.K., in 2010 and 2014, respectively. She was a Research Associate with the Department of Electronic and Electrical Engineering, University of Sheffield, from 2014 to 2017, and was responsible for fabrication and characterization of high sensitivity mid-infrared detectors and high-speed avalanche photodiodes. She has recently become a Senior Product Engineer of Detectors in Oclaro Technology Ltd, Caswell, U.K.

Alan P. Morrison (S'93-M'96-SM'02) received the Bachelor's degree (first class hons.) in electrical engineering and microelectronics from University College Cork, Cork, Ireland, in 1992, and the Ph.D. degree from the National University of Ireland, Galway, Ireland, in 1997.

$\mathrm{He}$ is currently the Dean of Engineering at University College Cork. His research interests include semiconductor optoelectronic devices, particularly optical detectors, photovoltaics, PV systems, and energy storage. He has authored and coauthored more than 120 papers in peer-reviewed journals and conferences. He is a member of the Institute of Physics and a Chartered Physicist.

Chee Hing Tan (M'95) received the B.Eng. and Ph.D. degrees in electronic engineering from the Department of Electronic and Electrical Engineering, University of Sheffield, Sheffield, U.K., in 1998 and 2002, respectively.

$\mathrm{He}$ is currently a Professor of Opto-Electronic Sensors with the Departmen of Electronic and Electrical Engineering, University of Sheffield. He has extensive experience in the characterization and modeling of high-speed low-noise avalanche photodiodes and phototransistors. His current research interests include single-photon avalanche diodes, mid-infrared photodiodes, quantum-dot infrared detectors, $\mathrm{X}$-ray detectors, ultrahigh-speed avalanche photodiodes, and phototransistors.

Jo Shien Ng (M'99) received the B.Eng. and Ph.D. degrees in electronic engineering from the University of Sheffield, Sheffield, U.K., in 1999 and 2003, respectively.

Between 2006 and 2016, she was a Royal Society Research Fellow with the Department of Electronic and Electrical Engineering, University of Sheffield, where she is currently a Professor of Semiconductor Devices. Her research interests include avalanche photodiodes, Geiger-mode avalanche photodiodes, and material characterization. 